\title{
Implications of HIF-1a in the tumorigenesis and progression of pancreatic cancer
}

\author{
Xiao Jin, Lu Dai, Yilan Ma, Jiayan Wang and Zheng Liu* (i)
}

\begin{abstract}
Pancreatic cancer is one of the leading causes of cancer-related deaths worldwide and is characterized by highly hypoxic tumor microenvironment. Hypoxia-inducible factor-1 alpha (HIF-1a) is a major regulator of cellular response to changes in oxygen concentration, supporting the adaptation of tumor cells to hypoxia in an oxygen-deficient tumor microenvironment. Numerous studies revealed the central role of HIF-1a in the carcinogenesis and progression of pancreatic cancer. This article reviewed the molecular mechanisms of how HIF-1 a regulated tumorigenesis and progression of pancreatic cancer and suggested that targeting HIF-1a and its signaling pathways could be promising therapeutics for pancreatic cancer.
\end{abstract}

Keywords: Hypoxia, HIF-1a, Pancreatic cancer, Tumorigenesis, Progression, Review

\section{Background}

According to the latest global cancer statistics in 2018, pancreatic cancer accounts for $2.5 \%$ of new cancers worldwide, and mortality accounts for $4.5 \%$ of all cancer deaths [1]. An assessment of tumor morbidity and mortality expects pancreatic cancer to rise to the second highest cancer mortality in the United States by 2030 [2]. At present, the high malignancy and poor curative effect of pancreatic cancer are mostly attributed to hypoxic tumor microenvironment $[3,4]$.

Hypoxia-inducible factor-1 (HIF-1) is a key factor regulating cell adaptation to hypoxia [5]. HIF-1 consists of an oxygen-regulated alpha subunit (HIF- $1 \alpha$ ) and a constitutively expressed beta subunit (HIF-1 $\beta$ ) [6]. Under normoxic conditions, the proline and lysine residues on the oxygen-dependent degradation domain of HIF- $1 \alpha$ are hydroxylated, and the modified HIF-1 $\alpha$ interacts with the Von Hippel-Lindau E3 ubiquitin ligase complex followed degradation through the ubiquitin-proteasome pathway [7]. However, HIF- $1 \alpha$ is stable in hypoxia and forms

*Correspondence: liuzheng117@126.com

Medical Center for Digestive Diseases, Second Affiliated Hospital, Nanjing

Medical University, 121 Jiangjiayuan Road, Nanjing 210011, Jiangsu, China heterodimers with HIF-1 $\beta$ with the help of coactivators such as cyclic adenosine monophosphate response element-binding protein (CBP) and acetyltransferase (p300), and then, HIF- $1 \alpha$ transfers to the nucleus and binds to the target gene hypoxia response element (HRE), a DNA sequence consisting of consecutive transcription factor binding sites that contains the core sequence of $5^{\prime}$-TAC GTG-3' (Fig. 1), modulating the targets transcription [5, 8]. In addition, HIF- $1 \alpha$ can also be activated by an oxygen-independent mechanism [9] (Fig. 2).

Pancreatic cancer possesses hypoxic niche and is accompanied by HIF-1 $\alpha$ overexpression [10, 11]. Increasing studies explore the roles of HIF- $1 \alpha$ in pancreatic cancer and pancreas embryonic development. This review mainly elucidated the major function of HIF- $1 \alpha$ in the carcinogenesis and progression of pancreatic cancer as well as pancreas embryonic development. Therefore, targeting HIF- $1 \alpha$ and its signaling pathways might be effective therapeutic approaches for pancreatic cancer.

\section{HIF-1 $\alpha$ in pancreas embryonic development and homeostasis}

Pancreatic blood flow is low and cells are hypoxic during the early stages of embryogenesis. Later, increasing oxygen concentration facilitates pancreatic cells further 


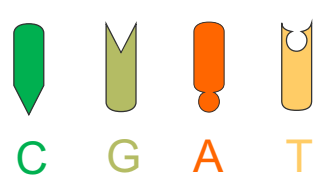

HRE

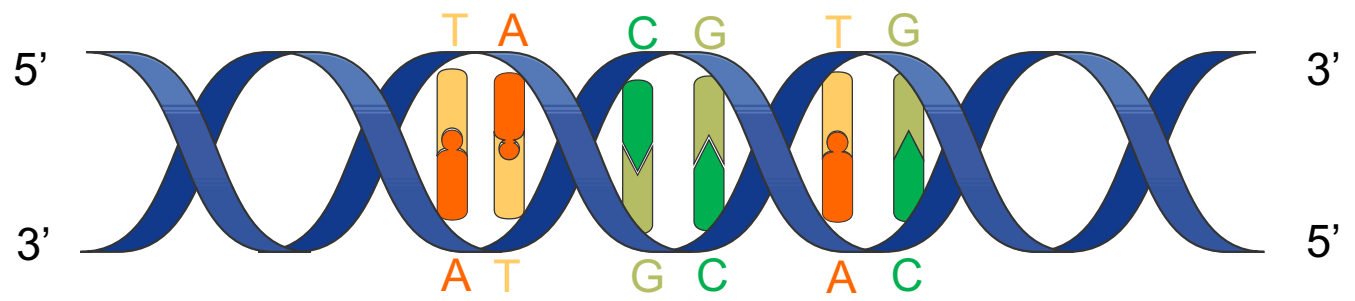

Fig. 1 HRE core sequence

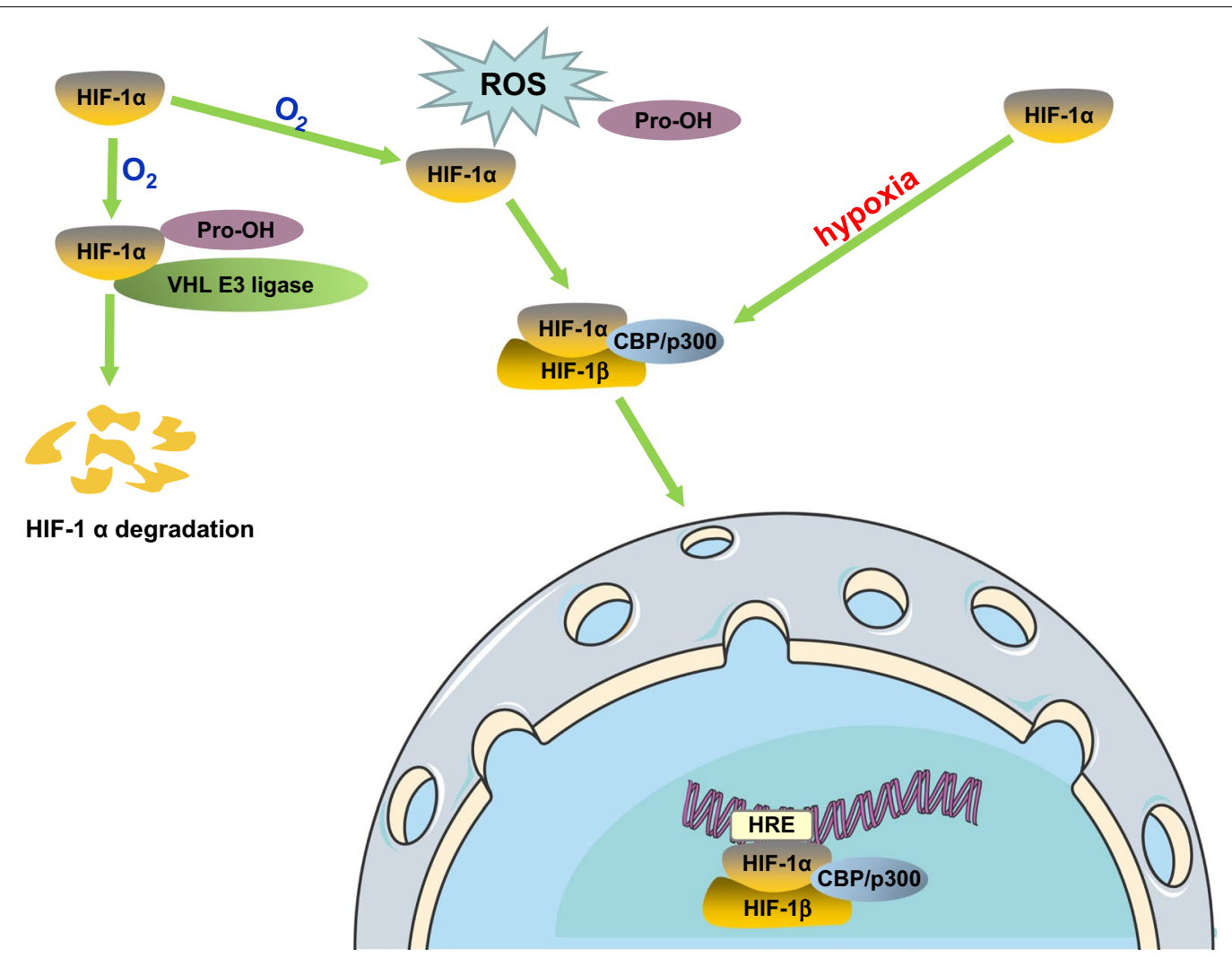

Fig. 2 HIF-1a degradation and activation. $\uparrow$, promote. Under normoxia, HIF-1a is hydroxylated by prolyl hydroxylases and binds to VHL which recruits E3-ubiquitin ligase to interact with $\mathrm{HIF-1}$ a, resulting in degradation of HIF-1a in a ubiquitin-proteasome way. Besides, the existence of ROS in normoxia inhibits the acetylation of HIF-1 a via blocking the activation of PHDs, protecting HIF-1a from degradation. In hypoxia, oxygen deficiency inhibits hydroxylation of HIF-1a, HIF-1a forms heterodimers with HIF-1 $\beta$ with help of CBP/p300 and transfers to the nucleus to bind to its target genes 
differentiation [12]. HIF-1 $\alpha$ level gradually decreases and plays a central role in responding to changes in oxygen during pancreas embryonic development [13]. Low expression of HIF- $1 \alpha$ in islets regulated glucose-stimulated insulin secretion and protected $\beta$-cells reserve and function via binding to HRE in the promoter of aryl hydrocarbon receptor nuclear translocator (ARNT), but deletion of HIF- $1 \alpha$ impaired $\beta$-cells function [14]. Congruously, appropriate level of HIF- $1 \alpha$-mediated vascular endothelial growth factor A (VEGF-A) expression contributed to normal pancreatic growth and development of islet-specific capillary fenestrations, maintaining islet $\beta$-cells mass and function [15]. However, high expression of HIF- $1 \alpha$ lost the function of maintaining pancreas development and its homeostasis. Studies suggested overexpressed HIF- $1 \alpha$ inhibited the differentiation of pancreatic endocrine progenitor cells via suppressing the expression of neurogenin3, a pro-endocrine transcription factor, through activating mTOR complex I signaling, and suppressed islet $\beta$-cells differentiation via mediating hypervascularization $[13,16,17]$. Besides, data indicated diabetes and higher blood glucose levels among those without diabetes were potential risks of pancreatic cancer, hyperglycemiainduced HIF- $1 \alpha$ overexpression and microenvironment hypoxia, upregulating MMP-9 expression and promoting pancreatic cancer progression in a HIF- $1 \alpha$-dependent manner. Hyperglycemia was mostly attributed to $\beta$-cells dysfunction, while low level of HIF- $1 \alpha$ was required for $\beta$-cells function maintenance. This might imply that the different roles of HIF-1 $\alpha$ in development and carcinogenesis of pancreas depended on the difference in its expression levels $[18,19]$. Surprisingly, upregulation of HIF- $1 \alpha$ does not necessarily exert pathogenic or carcinogenic effects in pancreas. HIF- $1 \alpha$ acted as a downstream molecule of mTOR and regulated glucagon-like peptide-1 (GLP-1) receptor-induced metabolism reprogramming via PI3K/mTOR pathway, enhancing mice islet viability $[20,21]$. Moreover, HIF-1 $\alpha$ accumulation contributed to pancreas tissue regeneration via inhibiting intrapancreatic B lymphocytes accumulation in cerulein-induced experimental mice pancreatitis [22]. In addition, islets cultured in vitro tended to lose their heavy vascularization, but hypoxia-induced HIF- $1 \alpha$ could maintain this vasculature via enhancing vascular length and endothelial cells area through upregulating VEGF-A expression, facilitating transplantation survival [23]. Additionally, the protective effect of exendin-4, a GLP-1 receptor agonist, for transplantation islets during hypoxic phase was also attributed to overexpression of HIF-1 $\alpha$ [24]. However, current researches have consistently revealed that HIF- $1 \alpha$ is overexpressed in patients with pancreatic cancer and regulated various genes expression (Table 1), acting as an oncogene in pancreatic cancer $[10,11]$.

\section{HIF-1a and pancreatic cancer parenchyma Survival and proliferation}

Aberrant cells proliferation is the most basic feature in the tumorigenesis. Studies indicate HIF- $1 \alpha$ promotes the proliferation of pancreatic cancer cells through various mechanisms. IncRNA is involved in the modulation of HIF-1 $\alpha$ in the carcinogenesis [25]. In pancreatic cancer, HIF- $1 \alpha$ bound to the HRE of IncRNA-NUTF2P3-001, upregulating its expression. Overexpressed lncRNANUTF2P3-001 competitively bound to miR-3923, increasing V-Ki-ras2 Kirsten Rat Sarcoma Viral Oncogene Homolog (KRAS) expression, which resulted in significant improvement of tumor cells survival rate and proliferation [26]. Besides, lncRNA-FEZF1-AS1 enhanced pancreatic cancer cells proliferation via the miR-142/HIF- $1 \alpha$ axis in hypoxia [27]. Moreover, decreased IncRNA-CF129 regulated by HIF-1 $\alpha /$ histone deacetylase 1(HDAC1) complex facilitated pancreatic cancer progression via increasing forkhead box $\mathrm{C} 2$ (FOXC2) expression, and FOXC2 overexpression was induced by E3 ligase MKRN1-mediated p53 ubiquitin degradation. Furthermore, FOXC2 and HIF- $1 \alpha$ regulated reciprocally and formed a positive feedback loop [28]. Stromal interaction molecule 1 (STIM1) is regulated by HIF- $1 \alpha$ and is implicated in the cells proliferation. The study indicated STIM1 expression was upregulated, accompanied by HIF- $1 \alpha$ overexpression in pancreatic cancer tissues. HIF- $1 \alpha$ bound to the HRE of STIM1 and elevated its expression, thereby increasing the proliferation of pancreatic cancer cells [10].

\section{Metabolism reprogramming}

High metabolism is a notable property of tumor cells. Aerobic glycolysis pathway can produce large amounts of adenosine triphosphate in a short period of time and is one of the most important metabolic modes of tumor cells [29]. HIF-1 $\alpha$ is considered as a primary conditioner of metabolism reprogramming [30]. Decreased Rho GTPase-activating protein 4 (ARHGAP4) facilitates aerobic glycolysis of pancreatic cancer through activating HIF- $1 \alpha$ pathway and upregulating M2 isoform of pyruvate kinase (PKM2) expression [31]. Mucin1 is a type I transmembrane protein which is widely expressed in pancreatic cancer tissues and regulates anabolic glucose metabolism in a HIF-1 $\alpha$ dependent manner in pancreatic cancer [32]. Mucin1 enhanced the stability of HIF- $1 \alpha$ and recruited HIF- $1 \alpha$ and p300 to bind to the promoter of glycolytic genes such as enolase1 (ENO1) and phosphoglucomutase-2 
Table 1 Genes induced by HIF-1a in pancreatic cancer tumorigenesis and progression

\begin{tabular}{|c|c|c|}
\hline Target genes & Acting mechanisms & References \\
\hline LncRNA-NUTF2P3-001 & 个LncRNA-NUTF2P3-001, $\downarrow$ miR-3923, KRAS, $\uparrow$ cells viability, proliferation and invasion & {$[26]$} \\
\hline LncRNA-CF129 & 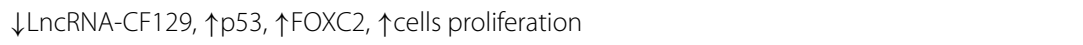 & {$[28]$} \\
\hline STIM1 & 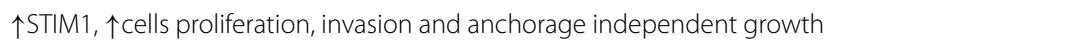 & {$[10]$} \\
\hline PKM2 & $\uparrow P K M 2, \uparrow g l y c o l y s i s$ & {$[31]$} \\
\hline ENO1, PGM2 & $\uparrow E N O 1$ and $P G M 2, \uparrow$ glycolysis & {$[33]$} \\
\hline GLUT1, LDHA, HK2 & 个GLUT1, LDHA and HK2, $\uparrow$ glycolysis & {$[37]$} \\
\hline CypA & $\uparrow C y p A, \downarrow$ apoptosis, $\uparrow$ cells proliferation, migration and invasion & {$[38]$} \\
\hline MiR-21 & $\uparrow M i R-21, \downarrow$ apoptosis, $\uparrow$ cells proliferation & {$[40]$} \\
\hline MT2-MMP & 个MT2-MMP, $\downarrow$ apoptosis, $\uparrow$ cells proliferation and invasion & {$[42]$} \\
\hline MTA2 & $\uparrow M T A 2, \downarrow E$-cadherin, $\uparrow E M T$, cells migration and invasion & {$[49]$} \\
\hline Twist & 个Twist, $\downarrow$ E-cadherin, $\uparrow$ EMT and cells proliferation & {$[50]$} \\
\hline Snail & 个Snail, $\downarrow$ E-cadherin, $\uparrow N$-cadherin, $\uparrow E M T$, cells migration and invasion & {$[51]$} \\
\hline LncRNA-BX111 & 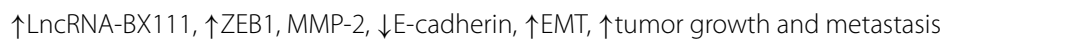 & {$[52]$} \\
\hline CD133 & $\uparrow C D 133, \uparrow$ tumor stem cell properties, $\uparrow$ cells migration and invasion & {$[56]$} \\
\hline RER1 & $\begin{array}{l}\uparrow R E R 1, \uparrow N \text {-cadherin, Vimentin and Snail, } \downarrow \text { E-cadherin, } \uparrow \text { Sox2, Bmi1, Lin28 and Nanog, } \uparrow E M T \text { and } \\
\text { cancer stem cell-like properties }\end{array}$ & {$[58]$} \\
\hline ATG5, Beclin 1 & 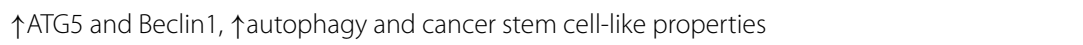 & {$[59]$} \\
\hline ABCG2 & $\uparrow A B C G 2, \uparrow$ chemoresistance & {$[64]$} \\
\hline$N F-k B$ & $\uparrow N F-k B, \downarrow E$-cadherin, $\uparrow N$-cadherin, Vimentin, Snail, Twist, $\uparrow E M T$ and chemoresistance & {$[65]$} \\
\hline CXCR4 & $\uparrow C X C R 4, \uparrow$ chemoresistance & {$[66]$} \\
\hline IL-37 & $\downarrow \mathbb{I L}-37, \uparrow$ chemoresistance & {$[68]$} \\
\hline VEGF-A & 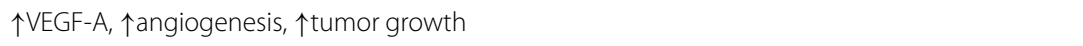 & [73] \\
\hline STAT3, VEGF-A & 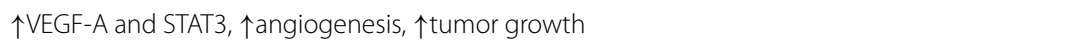 & {$[75]$} \\
\hline CHC, VEGF-A & $\uparrow C H C$ and VEGF-A, $\uparrow$ angiogenesis, $\uparrow$ tumor growth & {$[77]$} \\
\hline VEGF, MMP-2, MMP-9 & 个VEGF, MMP-2 and MMP-9, $\uparrow$ angiogenesis, $\uparrow$ tumor growth & {$[78]$} \\
\hline ADAM10 & $\uparrow A M A D 10, \downarrow m M I C A, \uparrow s M I C A, \downarrow N K G 2 D, \downarrow N K$ cells cytotoxicity, $\uparrow$ immune evasion & {$[82]$} \\
\hline $\mathrm{CCL} 2$ & $\uparrow C C L 2, \uparrow a-S M A, \uparrow$ desmoplasia & {$[85]$} \\
\hline $\mathrm{SHH}$ & $\uparrow S H H, \uparrow H H$ signaling, $\uparrow$ collagen I and fibronectin, $\uparrow$ desmoplasia & {$[84,86]$} \\
\hline
\end{tabular}

$\uparrow$, promote; $\downarrow$, inhibit; KRAS, V-Ki-ras2 Kirsten Rat Sarcoma Viral Oncogene Homolog; FOXC2, forkhead box C2; STIM1, stromal interaction molecule 1; PKM2, M2 isoform of pyruvate kinase; ENO1, enolase 1; PGM2, phosphoglucomutase-2; GLUT1, glucose transporter type 1; LDHA, lactate dehydrogenase A; HK2, hexokinase 2; CypA, cyclophilin A; MT2-MMP, membrane type-2 matrix metalloproteinase; MTA2, metastasis-associated protein 2; EMT, epithelial mesenchymal transition; ZEB1, zinc finger E-box-binding protein 1; MMP-2, matrix metalloproteinase 2; RER1, retention in endoplasmic reticulum 1; ATG5, autophagy related 5; ABCG2, ATP-binding cassette subfamily G member 2; CXCR4, chemokine receptor 4; VEGF-A, vascular endothelial growth factor A; STAT3, signal transducer and activator of transcription 3; CHC, clathrin heavy chain; MMP-9: matrix metalloproteinase 9; ADAM10, a disintegrin and metalloproteinase domain 10; mMICA, membrane major histocompatibility complex class I molecular associated proteins A; sMICA, soluble major histocompatibility complex class I molecular associated proteins A; NKG2D, natural killer group 2 member D; CCL2, chemical chemokine 2; a-SMA, a-smooth muscle actin; SHH, sonic hedgehog; $\mathrm{HH}$, hedgehog

(PGM2), upregulating their expression which contributed to increased glucose uptake and lactate production in pancreatic cancer cells [33]. Moreover, mucin1mediated HIF-1 $\alpha$ stability enhanced gemcitabine resistance via increased glucose metabolism [34]. Similarly, several studies confirmed that HIF-1 $\alpha$ increased expression of glycolytic-related enzymes and the production of lactic acid, meeting the metabolic needs of pancreatic cancer cells [34, 35]. Besides, the transcription factor 7-like 2 (TCF7L2) is correlated with the glycolysis in tumor cells [36]. The research indicated upregulation of TCF7L2 inhibited the promoter activity of Egl-9 family hypoxia-inducible factor 2 (EGLN2) and suppressed its expression, which enhanced the stability of HIF-1 $\alpha$, enhancing glycolysis-related genes expression and increasing glycolysis in pancreatic cancer cells [37].

\section{Anti-apoptosis and autophagy}

Numbers of studies revealed high expression of HIF- $1 \alpha$ markedly enhanced the anti-apoptotic capacity of pancreatic cancer cells $[34,35]$. In addition, research showed HIF-1 $\alpha$ bound directly to the HRE of cyclophilin A (CypA) and upregulated its expression, which inhibited pancreatic cancer cells apoptosis [38]. Besides, miR-21 is associated with tumor cells evading apoptosis [39]. 
In pancreatic cancer, HIF-1a induced miR-21 overexpression, preventing tumor cells from apoptosis in an oxygen-deficient environment [40]. Membrane type-2 matrix metalloproteinase (MT2-MMP), one of the members of the matrix metalloproteinase family, is expressed in tumor cells and is implicated in proliferation, migration and invasion of them [41]. Recent researches showed MT2-MMP was a new component in an anti-apoptotic pathway network in tumor cells and was also a novel target of HIF- $1 \alpha$. HIF- $1 \alpha$ bound to the HRE of MT2-MMP and activated its transcription, overexpressing MT2MMP clearly mitigated the apoptosis of pancreatic cancer cells [42]. Nevertheless, the roles of HIF-1 $\alpha$ in the apoptosis of pancreatic cancer cells remain controversial. Dai et al. reported that HIF- $1 \alpha$ could induce cells apoptosis in pancreatic cancer [43]. Therefore, the involvement of HIF- $1 \alpha$ in the regulation of apoptosis in pancreatic cancer in hypoxia requires further exploration.

It is well known that hypoxia-induced autophagy could promote tumor progression [44]. Autophagy is a metabolic pathway in which cells transport their proteins and organelles to lysosomal degradation, reducing their oxidative stress [45]. The study indicated that HIF-1 $\alpha$ induced autophagy potentiated epithelial mesenchymal transition (EMT) and migration of pancreatic cancer stem cells, increasing the malignancy of pancreatic cancer [46]. Besides, HIF-1 $\alpha$-mediated autophagy reduced lumican level secreted by pancreatic stellate cells, promoting pancreatic cancer progression [47].

\section{$E M T$, invasion and metastasis}

A large number of studies demonstrated HIF- $1 \alpha-$ mediated pancreatic cancer cells EMT, invasion and metastasis in hypoxia. Current researches focus on HIF- $1 \alpha$ regulating EMT processes via affecting EMTrelated proteins expression. Epithelial cell cadherin (E-cadherin) is a single transmembrane glycoprotein encoded by the cadherin 1 gene that maintains epithelial cell polarity and cell-to-cell contact. Deletion of E-cadherin induces EMT, relating to invasion and metastasis in cancers [48]. HIF-1 $\alpha$ could inhibit E-cadherin expression via recruiting metastasis-associated protein 2 (MTA2)/ HDAC1 complex to bind to E-cadherin promoter, inducing EMT in pancreatic cancer cells [49]. Besides, the transcription of E-cadherin is also regulated by Twist, which is regulated by HIF- $1 \alpha$ in hypoxia and serves as an important transcription factor promoting EMT of tumor cells. In pancreatic cancer, Twist recruited Ring1B and enhancer of zeste homolog 2 (EZH2), members of polycomb family, to bind to the promoter of E-cadherin and suppressed its transcription, inducing EMT [50]. Moreover, HIF- $1 \alpha$ increased Snail transcription through binding to its HRE, contributing to EMT in pancreatic cancer [51]. Furthermore, numerous studies showed lncRNAs were involved in the regulation of EMT. Deng et al. reported that HIF- $1 \alpha$ and lncRNA-BX111887 were overexpressed in pancreatic cancer tissues. Under the mediation of HIF- $1 \alpha$, lncRNA-BX111887 recruited transcriptional factor Y-box protein to the promoter of zinc finger E-box-binding protein 1 (ZEB1), a major factor inducing EMT, and promoted its transcription [52]. Additionally, miRNAs are implicated in the regulation of HIF- $1 \alpha$ on the EMT of pancreatic cancer cells. MiR142 expression in pancreatic cancer tissues and pancreatic cancer cell lines was significantly lower than that in normal tissues. Down-regulated miR-142 increased the expression of HIF-1 $\alpha$, upregulating EMT-related proteins, enhancing the invasion and migration of pancreatic cancer cells [53].

\section{Tumor stem cells}

It is well known that tumor stem cells are the key to tumor cells clone formation, proliferation and migration, which are not easily eliminated by anti-tumor drugs and can further differentiate as well as support tumor development [54]. One of the reasons for pancreatic cancer recrudescence and treatment resistance is the presence of tumor stem cells [55]. CD133 is one of surface molecules of pancreatic cancer stem cells, and its expression is increased in a HIF-1 $\alpha$-dependent manner in hypoxia [56]. Moreover, CD133 elevated HIF- $1 \alpha$ transcriptional activity in pancreatic cancer cells in hypoxia, initiating its expression and activating its target genes, which in turn induced EMT phenotype and tumor cells migration [57]. Besides, the study indicated HIF- $1 \alpha$ bound to the regulatory region in the upstream of the initiation codon of retention in endoplasmic reticulum 1 (RER1) and increased its transcriptional activity, enhancing pancreatic cancer stem cells properties and inducing EMT [58]. Additionally, the study elucidated that HIF-1 $\alpha$ induced autophagy mediated the conversion of non-stem pancreatic cancer cells to pancreatic cancer stem cells in hypoxia, enhancing the malignancy of pancreatic cancer [59].

\section{Chemoresistance and radiotherapy resistance}

Most pancreatic cancer patients are diagnosed in the advanced stages or even with distant metastasis due to absence of early recognizable symptoms, missing the opportunity for radical surgical resection. Thus, chemotherapy and radiotherapy have become the main therapies for pancreatic cancers currently, but the efficacy of chemotherapy and radiotherapy are limited by factors inside and outside the tumor cells $[60,61]$. The study suggested hypoxia-induced HIF- $1 \alpha$ contributed to chemoresistance and radiotherapy resistance in pancreatic 
cancer [62]. ATP-binding cassette subfamily G member 2 (ABCG2) is a multidrug resistant pump that is associated with drug resistance in numerous malignancies [63]. In pancreatic cancer, hypoxia-induced phosphorylation of ERK $1 / 2$ activated HIF- $1 \alpha$, increasing the accumulation of HIF- $1 \alpha$ in the cytoplasm and translocating to the nucleus, and then bound to the HRE of ABCG2 and promoted its transcription, thereby enhancing the drug resistance [64]. Several studies revealed that HIF- $1 \alpha$ and NF- $\kappa B$ expression were increased in pancreatic cancer tissues and there was a positive feedback regulation between them, resulting in chemoresistance partly. In pancreatic cancer, gemcitabine treatment activated NF- $\mathrm{KB}$ and HIF- $1 \alpha$ via ROS-mediated activation of ERK1/2 and Akt, upregulating chemokine receptor 4 (CXCR4) expression and contributing to gemcitabine resistance by CXCR4/chemokine 12 (CXCL12) signaling $[65,66]$. Besides, hypoxia-induced miR-301a overexpression induced gemcitabine resistance via enhancing HIF- $1 \alpha$ accumulation through suppressing expression of TAp63 which could down-regulate HIF- $1 \alpha$ expression through proteasomal degradation [67]. IL-37 expression was decreased in pancreatic cancer, down-regulated IL37-mediated gemcitabine resistance via interacting with HIF- $1 \alpha$ and signal transducer and activator of transcription 3 (STAT3) and forming the HIF-1 $\alpha /$ IL-37/STAT3 negative feedback [68]. Heat shock protein90 (HSP90) is a pivotal molecular chaperone of HIF- $1 \alpha$, which plays a crucial role in the correct folding, stability and transcription of HIF-1 $\alpha$. Inhibiting HSP90 could reverse HIF-1 $\alpha$ mediated resistance to radiotherapy and chemotherapy in pancreatic cancer $[69,70]$.

\section{HIF-1 $\alpha$ and pancreatic cancer stroma Angiogenesis}

Tumor angiogenesis is known to be essential for growth and metastasis of pancreatic cancer [71]. VEGF regulated by HIF- $1 \alpha$ at transcription level is essential to angiogenesis [72]. Azoitei et al. reported VEGF-A was not only a target of HIF- $1 \alpha$, but also of NF- $\kappa B$ transcription factor, involved in regulating tumor progression. In pancreatic cancer, PKM2 upregulated HIF-1 $\alpha$ in a NF- $\mathrm{kB} /$ p65-dependent manner, inducing VEGF-A expression which contributed to tumor angiogenesis and growth [73]. The study showed both STAT3 and HIF- $1 \alpha$ were client proteins of HSP90 [74]. In pancreatic cancer, HSP90 promoted VEGF-mediated angiogenesis via activating IL-6/HIF-1 $\alpha /$ STAT3 autocrine loop [75]. Serine/ threonine kinase 33 (STK33), a serine/threonine kinase, is a new client protein of HSP90. STK33 participated in tumor angiogenesis promoted by HSP90 chaperone via upregulating HIF- $1 \alpha$ accumulation and its target gene VEGF secretion in pancreatic cancer [76]. Clathrin is a trimer of heavy chains, each paired with a light chain. Clathrin heavy chain (CHC) interacted with HIF- $1 \alpha$ and co-localized to the HRE in the VEGF-A promoter region, upregulating VEGF-A expression to increase angiogenesis in pancreatic cancer [77]. Intriguingly, HIF-1 $\alpha$ could also mediate stress-induced pancreatic tumor growth and angiogenesis via regulating the expression of VEGF, matrix metalloproteinase 2 (MMP-2) and matrix metalloproteinase 9 (MMP-9) [78].

\section{Immune evasion}

The surfaces of tumor cells generally express new antigens, which are recognized by immune system, subsequently activating innate and acquired immune responses. However, tumor cells could evade immune responses via modifying the surface antigens or altering the tumor microenvironment [79]. Major histocompatibility complex class I molecular-associated proteins A (MICA) and major histocompatibility complex class I molecular-associated proteins B (MICB) are highly expressed on the various tumor cells membrane, acting as ligands of natural killer group 2 member D (NKG2D) expressed on NK cells and $\gamma \delta \mathrm{T}$ cells. NKG2D interacts with its ligand for immune surveillance and lysis of tumor cells, and this process is regulated by HIF- $1 \alpha[80,81]$. In pancreatic cancer, HIF- $1 \alpha$ and ADAM10, a disintegrin and metalloproteinase domain 10 (ADAM10) are highly expressed and are negatively regulated by miR-153. Overexpressed circ-0000977 serves as a sponge for miR-153 to counteract miR-153-mediated suppression of HIF-1 $\alpha$ and ADAM10, promoting the shedding of membrane MICA (mMICA) from surface of tumor cells and converting into soluble MICA (sMICA). SMICA binds to NKG2D, down-regulating NKG2D expression as well as cytotoxicity of NK cells, resulting in immune evasion [82].

\section{Desmoplasia}

Pancreatic cancer is characterized by desmoplasia and highly hypoxic microenvironment composed of tumor cells, extracellular matrix, fibroblasts, endothelial cells and immune cells, the both amplify each other in a positive feed-back loop and accelerate pancreatic cancer progression [83, 84]. In hypoxic microenvironment, hypoxia-induced chemokines attract monocytes/macrophages to inflammation, damage tissues and tumor tissues. In pancreatic cancer, hypoxia-induced HIF- $1 \alpha$ bound to the HRE of chemical chemokine 2 (CCL2) and increased its expression, recruiting macrophages to infiltrate tumor tissues to activate pancreatic stellate cells and confer it fibroblast phenotypes via increasing the expression of $\alpha$-smooth muscle actin, aggravating the hypoxic microenvironment of pancreatic cancer and accelerating its progression [85]. Studies indicated sonic hedgehog 
(SHH) ligand was conducive to occurrence of desmoplasia, and paracrine hedgehog $(\mathrm{HH})$ signaling played a central role in tumorigenic communication between tumor cells and fibroblasts in the stroma in pancreatic cancer. Hypoxia increased the expression of SHH in a HIF- $1 \alpha-$ dependent manner, and its overexpression activated $\mathrm{HH}$ signaling and collagen I fibronectin formation, inducing desmoplasia and enhancing tumor aggressiveness in pancreatic cancer $[84,86]$.

\section{Prospective into clinical application}

HIF- $1 \alpha$ manipulates the malignant biological features of pancreatic cancer through various pathways. Hence, targeting HIF- $1 \alpha$ and its signaling pathways could be potential therapeutics for pancreatic cancer. It is difficult to block HIF- $1 \alpha$ directly because it is a transcription factor and mainly locates in the nucleus [5]. Currently, promoting the degradation of HIF- $1 \alpha$ protein and targeting certain molecules in the HIF- $1 \alpha$ signaling pathways are effective therapeutics for pancreatic cancer. As is known to all, HIF- $1 \alpha$ is degraded via the ubiquitin-mediated proteasome-dependent pathway under normoxia and is stable in hypoxia. Nevertheless, infection of pancreatic cancer cells with oncotropic $\mathrm{H}-1$ parvovirus could rapidly degrade HIF-1 $\alpha$ in a proteasome-dependent manner in hypoxia, and this process was independent of Von Hippel-Lindau and receptor of activated protein $C$ kinase [87]. Analogously, pharmacologic ascorbate (P-AscH) rapidly degrades HIF- $1 \alpha$ in a proteasome-dependent pathway in pancreatic cancer cells which is independent of 2-oxoglutarate-dependent prolyl hydroxylase (PHD-2). $\mathrm{P}-\mathrm{AscH}$ can also increase extracellular $\mathrm{H}_{2} \mathrm{O}_{2}$ and transport it into tumor cells through the plasma membrane to inhibit the expression of HIF-1 $\alpha$ and VEGF, exerting a killing effect on pancreatic cancer cells [88]. Besides, extracellular superoxide dismutase could accelerate the degradation of HIF- $1 \alpha$ via reducing peroxides in pancreatic cancer [89]. The study showed xylene derivative TEL03 could bind to HIF- $1 \alpha$ to block the combination of HIF- $1 \alpha$ and $\mathrm{p} 300$, and induce the degradation of HIF- $1 \alpha$ by proteasome pathway in pancreatic cancer. Moreover, TEL03 could inhibit the phosphorylation of STAT3, acting as an upstream molecule and transcriptional factor of HIF- $1 \alpha$, suppressing HIF- $1 \alpha$ expression [90]. Notably, TX-2098, a hypoxia cytotoxin, directly down-regulated the protein level of HIF- $1 \alpha$ and its downstream targets such as VEGF, glucose transporter type 1 and aldolase A in pancreatic cancer, inhibiting its progression [91]. Prolyl hydroxylase domain 3 (PHD3), a rate-limiting enzyme regulating HIF- $1 \alpha$ degradation, improves radiotherapy efficacy through inhibiting p-EGFR/HIF- $1 \alpha$ signaling in pancreatic cancer [92].
In addition to the acceleration of HIF- $1 \alpha$ degradation, targeting the HIF- $1 \alpha$ signaling pathway is also a momentous avenue to impede the progression of pancreatic cancer. Alpha-solanine, a steroidal alkaloid with anti-tumor effects extracted from plants of the family Solanaceae, inhibits pancreatic cancer cells proliferation, migration and invasion through targeting p-ERK1/2-HIF-1 $\alpha$-VEGF axis [93, 94]. Similarly, HS-345, an inhibitor targeting tropomyosin-related kinase A, and HS-527, an inhibitor of PI3K, inhibit angiogenesis via targeting HIF-1 $\alpha /$ VEGF axis in pancreatic cancer $[95,96]$. Besides, curcumin, a natural polyphenol present in turmeric, and its analogues suppress the expression of HIF- $1 \alpha$ by inhibiting its interaction with HSP90 and NF- $\mathrm{KB}$ signaling pathway in pancreatic cancer [97]. Triptolidenol-1 (LB-1) is a derivative of $L B$, with less toxicity. In pancreatic cancer, LB-1 decreased the activity of HIF- $1 \alpha$ via inhibiting its upstream pathway PI3K/Akt/mTOR, moreover, LB-1 could inhibit the connection between HIF-1 $\alpha$, p-STAT3 and p300, repressing VEGF expression, in addition, LB-1 accelerated HIF- $1 \alpha$ degradation by ubiquitin-proteasome pathway [98]. Additionally, in pancreatic cancer, combination of PX-478, a specific agent suppressing constitutive and hypoxia-induced expression of HIF-1 $\alpha$, with gemcitabine induce immunogenic cell death which is related to repression of HIF-1 $\alpha$ via upregulating phosphorylation of eIF2 $\alpha$ [99], PX-478 can enhance radiosensitization by inhibition of HIF- $1 \alpha$ as well [100].

Notable is, studies indicate that two types of hypoxia are present in tumor, chronic and cycling hypoxia. Cycling hypoxia more appropriately describes the dynamic changes of hypoxia and reoxygenation in tumor and has been demonstrated to induce tumor aggressiveness more significantly than chronic hypoxia [101-103]. At present, the hypoxia status in most studies is set to chronic hypoxia, and the duration of hypoxia and the oxygen concentration are not consistent among the studies, which cannot completely simulate the pancreatic cancer hypoxic microenvironment in vivo, causing experimental results instability potentially. Besides, each study selects specific pancreatic cancer cell lines, and the molecular mechanisms proven in these studies might not be applied to all pancreatic cancer cells, which limit their application to the clinical treatment of pancreatic cancer possibly. HIF- $1 \alpha$ is in a complex signaling network and regulates the biological characteristics of pancreatic cancer cells in diverse manners. Therefore, future studies should focus on the entire signal network and explore central regulatory molecules to effectively inhibit pancreatic cancer progression. Our manuscript integrates acting mechanisms 


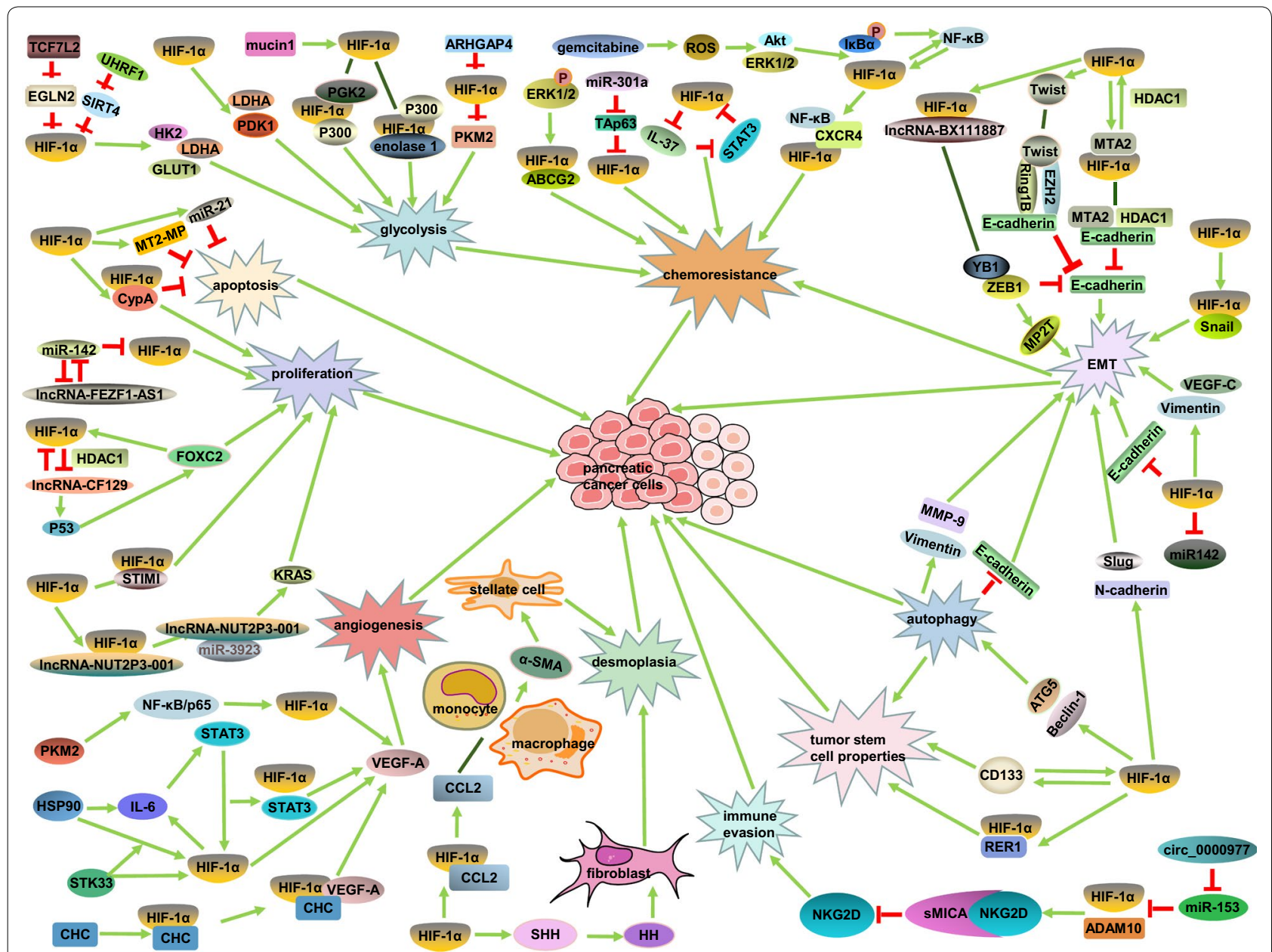

Fig. 3 Signaling pathways induced by HIF-1a in pancreatic cancer tumorigenesis and progression. $\uparrow$, promote; T, inhibit; I, recruit

of HIF- $1 \alpha$ to provide a comprehensive perspective of the role of HIF- $1 \alpha$ in pancreatic cancer, showing theoretical principle for the targeted therapy of pancreatic cancer.

\section{Conclusions}

As shown in Fig. 3 in pancreatic cancer hypoxic microenvironment, HIF- $1 \alpha$ induces tumor cells malignant biological characteristics and mediates tumorigenic crosstalk between tumor parenchyma and stroma. This suggests novel therapeutic strategies targeting HIF- $1 \alpha$ and its signaling pathways might be promising for pancreatic cancer therapy.

\section{Abbreviations}

HIF-1: Hypoxia-inducible factor-1; HIF-1 a: Hypoxia-inducible factor-1 alpha subunit; HIF-1 $\beta$ : Hypoxia-inducible factor-1 beta subunit; CBP: Cyclic adenosine monophosphate response element-binding protein; P300: Acetyltransferase: HRE: Hypoxia response element; ARNT: Aryl hydrocarbon receptor nuclear translocator; VEGF-A: Vascular endothelial growth factor A; KRAS: V-Ki-ras2 Kirsten Rat Sarcoma Viral Oncogene Homolog; HDAC1: Histone deacetylase
1; FOXC2: Forkhead box C2; STIM1: Stromal interaction molecule 1; ARHGAP4: Rho GTPase-activating protein 4; PKM2: M2 isoform of pyruvate kinase; ENO1: Enolase1; PGM2: Phosphoglucomutase-2; TCF7L2: Transcription factor 7-like 2; EGLN2: Egl-9 family hypoxia-inducible factor 2; CypA: Cyclophilin A; MT2-MMP: Membrane type-2 matrix metalloproteinase; EMT: Epithelial mesenchymal transition; E-cadherin: Epithelial cell cadherin; MTA2: Metastasis-associated protein 2; EZH2: Enhancer of zeste homolog 2; ZEB1: Zinc finger E-box-binding protein 1; RER1: Retention in endoplasmic reticulum 1; ABCG2: ATP-binding cassette subfamily G member 2; CXCR4: Chemokine receptor 4; CXCL12: Chemokines 12; STAT3: Signal transducer and activator of transcription 3; HSP90: Heat shock protein90; STK33: Serine/threonine kinase 33; CHC: Clathrin heavy chain; MMP-2: Matrix metalloproteinase 2; MMP-9: Matrix metalloproteinase 9; MICA: Major histocompatibility complex class I molecular-associated proteins A; MICB: Major histocompatibility complex class I molecular-associated proteins B; NKG2D: Natural killer group 2 member D; ADAM10: A disintegrin and metalloproteinase domain 10; SMICA: Soluble MCIA; SMICB: Soluble MCIB; CCL2: Chemical chemokine 2; SHH: Sonic hedgehog; HH: Hedgehog; P-AsCH: Pharmacologic ascorbate: PHD-2: 2-oxoglutarate-dependent prolyl hydroxylase; PHD3: Prolyl hydroxylase domain 3; LB-1: Triptolidenol-1.

\section{Acknowledgements}

Not applicable.

Authors' contributions

$\mathrm{XJ}$ wrote the paper; XJ, LD, YLM, JYW, and ZL revised the whole paper. All authors read and approved the final manuscript. 


\section{Funding}

Not applicable.

\section{Availability of data and materials}

All data are included in this article.

\section{Ethics approval and consent to participate}

Not applicable.

\section{Consent for publication}

Not applicable.

\section{Competing interests}

The authors declare that they have no competing interests.

Received: 31 March 2020 Accepted: 19 June 2020

Published online: 24 June 2020

\section{References}

1. Bray F, Ferlay J, Soerjomataram I, Siegel RL, Torre LA, Jemal A. Global cancer statistics 2018: GLOBOCAN estimates of incidence and mortality worldwide for 36 cancers in 185 countries. CA Cancer J Clin. 2018;68(6):394-424

2. Rahib $L$, Smith BD, Aizenberg R, Rosenzweig AB, Fleshman JM, Matrisian LM. Projecting cancer incidence and deaths to 2030: the unexpected burden of thyroid, liver, and pancreas cancers in the United States. Cancer Res. 2014;74(11):2913-21.

3. Koong AC, Mehta VK, Le QT, Fisher GA, Terris DJ, Brown JM, et al. Pancreatic tumors show high levels of hypoxia. Int J Radiat Oncol Biol Phys. 2000;48(4):919-22.

4. Dauer P, Nomura A, Saluja A, Banerjee S. Microenvironment in determining chemo-resistance in pancreatic cancer: neighborhood matters. Pancreatology. 2017;17(1):7-12.

5. Freedman SJ, Sun ZY, Poy F, Kung AL, Livingston DM, Wagner G, et al. Structural basis for recruitment of CBP/p300 by hypoxia-inducible factor-1 alpha. Proc Natl Acad Sci USA. 2002;99(8):5367-72.

6. Harris AL. Hypoxia-a key regulatory factor in tumour growth. Nat Rev Cancer. 2002;2(1):38-47.

7. Pereira T, Zheng $X$, Poellinger L. Degradation of the hypoxia-inducible factor 1alpha: where does it happen? Cell Cycle (Georgetown, Tex). 2006;5(23):2720-2.

8. Wu D, Potluri N, Lu J, Kim Y, Rastinejad F. Structural integration in hypoxia-inducible factors. Nature. 2015;524(7565):303-8.

9. Lee BL, Kim WH, Jung J, Cho SJ, Park JW, Kim J, et al. A hypoxiaindependent up-regulation of hypoxia-inducible factor-1 by AKT contributes to angiogenesis in human gastric cancer. Carcinogenesis. 2008;29(1):44-51

10. Wang J, Shen J, Zhao K, Hu J, Dong J, Sun J. STIM1 overexpression in hypoxia microenvironment contributes to pancreatic carcinoma progression. Cancer Biol Med. 2019;16(1):100-8.

11. Ye LY, Zhang Q, Bai XL, Pankaj P, Hu QD, Liang TB. Hypoxia-inducible factor $1 \mathrm{a}$ expression and its clinical significance in pancreatic cancer: a meta-analysis. Pancreatology. 2014;14(5):391-7.

12. Shah SR, Esni F, Jakub A, Paredes J, Lath N, Malek M, et al. Embryonic mouse blood flow and oxygen correlate with early pancreatic differentiation. Dev Biol. 2011;349(2):342-9.

13. Soggia A, Ramond C, Akiyama H, Scharfmann R, Duvillie B. von HippelLindau gene disruption in mouse pancreatic progenitors and its consequences on endocrine differentiation in vivo: importance of HIF1-a and VEGF-A upregulation. Diabetologia. 2014;57(11):2348-56.

14. Cheng K, Ho K, Stokes R, Scott C, Lau SM, Hawthorne WJ, et al. Hypoxiainducible factor-1alpha regulates beta cell function in mouse and human islets. J Clin Investig. 2010;120(6):2171-83.

15. Román CL, Maiztegui B, Mencucci MV, Ahrtz L, Algañarás M, Del Zotto $H$, et al. Effects of islet neogenesis associated protein depend on vascular endothelial growth factor gene expression modulated by hypoxia-inducible factor 1-alpha. Peptides. 2019;117:170090.
16. Rachdi L, Aïello V, Duvillié B, Scharfmann R. L-leucine alters pancreatic $\beta$-cell differentiation and function via the mTor signaling pathway. Diabetes. 2012;61(2):409-17.

17. Heinis M, Soggia A, Bechetoille C, Simon MT, Peyssonnaux C, Rustin $\mathrm{P}$, et al. HIF1a and pancreatic $\beta$-cell development. FASEB J. 2012;26(7):2734-42.

18. Pang Y, Kartsonaki C, Guo Y, Bragg F, Yang L, Bian Z, et al. Diabetes, plasma glucose and incidence of pancreatic cancer: a prospective study of 0.5 million Chinese adults and a meta-analysis of 22 cohort studies. Int J Cancer. 2017;140(8):1781-8.

19. Li W, Liu H, Qian W, Cheng L, Yan B, Han L, et al. Hyperglycemia aggravates microenvironment hypoxia and promotes the metastatic ability of pancreatic cancer. Comput Struct Biotechnol J. 2018;16:479-87.

20. Carlessi R, Chen Y, Rowlands J, Cruzat VF, Keane KN, Egan L, et al. GLP-1 receptor signalling promotes $\beta$-cell glucose metabolism via mTORdependent HIF-1 a activation. Sci Rep. 2017;7(1):2661.

21. Van de Velde S, Hogan MF, Montminy M. mTOR links incretin signaling to HIF induction in pancreatic beta cells. Proc Natl Acad Sci USA. 2011;108(41):16876-82.

22. Lee KE, Spata M, Maduka R, Vonderheide RH, Simon MC. Hif1 a deletion limits tissue regeneration via aberrant B cell accumulation in experimental pancreatitis. Cell Rep. 2018;23(12):3457-64.

23. Sankar KS, Altamentova SM, Rocheleau JV. Hypoxia induction in cultured pancreatic islets enhances endothelial cell morphology and survival while maintaining beta-cell function. PLOS ONE. 2019;14(10):e0222424.

24. Jia X, Sharma A, Kumagai-Braesch M, Wernerson AM, Sörenby AK, Yamamoto S, et al. Exendin-4 increases the expression of hypoxiainducible factor-1a in rat islets and preserves the endocrine cell volume of both free and macroencapsulated islet grafts. Cell Transplant. 2012;21(6):1269-83.

25. Sun S, Xia C, Xu Y. HIF-1a induced IncRNA LINC00511 accelerates the colorectal cancer proliferation through positive feedback loop. Biomed Pharmacother Biomed Pharmacother. 2020;125:110014.

26. Li X, Deng SJ, Zhu S, Jin Y, Cui SP, Chen JY, et al. Hypoxia-induced IncRNA-NUTF2P3-001 contributes to tumorigenesis of pancreatic cancer by derepressing the miR-3923/KRAS pathway. Oncotarget. 2016;7(5):6000-14.

27. Ou ZL, Zhang M, Ji LD, Luo Z, Han T, Lu YB, et al. Long noncoding RNA FEZF1-AS1 predicts poor prognosis and modulates pancreatic cancer cell proliferation and invasion through miR-142/HIF-1alpha and miR133a/EGFR upon hypoxia/normoxia. J Cell Physiol. 2019;234:15407.

28. Liu M, Zhong J, Zeng Z, Huang K, Ye Z, Deng S, et al. Hypoxia-induced feedback of HIF-1a and IncRNA-CF129 contributes to pancreatic cancer progression through stabilization of p53 protein. Theranostics. 2019;9(16):4795-810.

29. Ganapathy-Kanniappan S. Molecular intricacies of aerobic glycolysis in cancer: current insights into the classic metabolic phenotype. Crit Rev Biochem Mol Biol. 2018;53(6):667-82.

30. Tirpe AA, Gulei D, Ciortea SM, Crivii C, Berindan-Neagoe I. Hypoxia: overview on hypoxia-mediated mechanisms with a focus on the role of HIF genes. Int J Mol Sci. 2019;20(24):6140.

31. Shen $Y$, Chen $G$, Zhuang L, Xu L, Lin J, Liu L. ARHGAP4 mediates the Warburg effect in pancreatic cancer through the mTOR and HIF-1a signaling pathways. Onco Targets Ther. 2019;12:5003-12.

32. Shukla SK, Purohit V, Mehla K, Gunda V, Chaika NV, Vernucci E, et al. MUC1 and HIF-1alpha signaling crosstalk induces anabolic glucose metabolism to impart gemcitabine resistance to pancreatic cancer. Cancer Cell. 2017;32(1):71-87.e7.

33. Chaika NV, Gebregiworgis T, Lewallen ME, Purohit V, Radhakrishnan $P$, Liu X, et al. MUC1 mucin stabilizes and activates hypoxia-inducible factor 1 alpha to regulate metabolism in pancreatic cancer. Proc Natl Acad Sci USA. 2012;109(34):13787-92.

34. Jiang Y, Wu GH, He GD, Zhuang QL, Xi QL, Zhang B, et al. The effect of silencing HIF-1a gene in BxPC-3 cell line on glycolysis-related gene expression, cell growth, invasion, and apoptosis. Nutr Cancer. 2015;67(8):1314-23.

35. He G, Jiang Y, Zhang B, Wu G. The effect of HIF-1a on glucose metabolism, growth and apoptosis of pancreatic cancerous cells. Asia Pacific J Clin Nutr. 2014;23(1):174-80. 
36. Malakar P, Stein I, Saragovi A, Winkler R, Stern-Ginossar N, Berger M, et al. Long noncoding RNA MALAT1 regulates cancer glucose metabolism by enhancing mTOR-mediated translation of TCF7L2. Cancer Res. 2019;79(10):2480-93.

37. Xiang J, Hu Q, Qin Y, Ji S, Xu W, Liu W, et al. TCF7L2 positively regulates aerobic glycolysis via the EGLN2/HIF-1alpha axis and indicates prognosis in pancreatic cancer. Cell Death Dis. 2018;9(3):321.

38. Zhang H, Chen J, Liu F, Gao C, Wang X, Zhao T, et al. CypA, a gene downstream of HIF-1alpha, promotes the development of PDAC. PLoS ONE. 2014;9(3):e92824.

39. Zhao Q, Chen S, Zhu Z, Yu L, Ren Y, Jiang M, et al. miR-21 promotes EGFinduced pancreatic cancer cell proliferation by targeting Spry2. Cell Death Dis. 2018;9(12):1157.

40. Mace TA, Collins AL, Wojcik SE, Croce CM, Lesinski GB, Bloomston M. Hypoxia induces the overexpression of microRNA-21 in pancreatic cancer cells. J Surg Res. 2013;184(2):855-60.

41. Ito E, Yana I, Fujita C, Irifune A, Takeda M, Madachi A, et al. The role of MT2-MMP in cancer progression. Biochem Biophys Res Commun. 2010;393(2):222-7.

42. Zhu S, Zhou Y, Wang L, Zhang J, Wu H, Xiong J, et al. Transcriptional upregulation of MT2-MMP in response to hypoxia is promoted by HIF1alpha in cancer cells. Mol Carcinog. 2011;50(10):770-80.

43. Dai ZJ, Gao J, Ma XB, Yan K, Liu XX, Kang HF, et al. Up-regulation of hypoxia inducible factor-1alpha by cobalt chloride correlates with proliferation and apoptosis in PC-2 cells. J Exp Clin Cancer Res CR. 2012;31:28

44. Zhang W, Yuan W, Song J, Wang S, Gu X. LncRNA CPS1-IT1 suppresses EMT and metastasis of colorectal cancer by inhibiting hypoxia-induced autophagy through inactivation of HIF-1a. Biochimie. 2018;144:21-7.

45. Vikram A, Anish R, Kumar A, Tripathi DN, Kaundal RK. Oxidative stress and autophagy in metabolism and longevity. Oxid Med Cell Longev. 2017:2017:3451528.

46. Zhu H, Wang D, Zhang L, Xie X, Wu Y, Liu Y, et al. Upregulation of autophagy by hypoxia-inducible factor-1alpha promotes EMT and metastatic ability of CD133 + pancreatic cancer stem-like cells during intermittent hypoxia. Oncol Rep. 2014;32(3):935-42.

47. Li X, Lee Y, Kang Y, Dai B, Perez MR, Pratt M, et al. Hypoxia-induced autophagy of stellate cells inhibits expression and secretion of lumican into microenvironment of pancreatic ductal adenocarcinoma. Cell Death Differ. 2019;26(2):382-93.

48. Kourtidis A, Lu R, Pence LJ, Anastasiadis PZ. A central role for cadherin signaling in cancer. Exp Cell Res. 2017;358(1):78-85.

49. Zhu S, Deng S, He C, Liu M, Chen H, Zeng Z, et al. Reciprocal loop of hypoxia-inducible factor-1alpha (HIF-1alpha) and metastasis-associated protein 2 (MTA2) contributes to the progression of pancreatic carcinoma by suppressing E-cadherin transcription. J Pathol. 2018;245(3):349-60

50. Chen S, Chen JZ, Zhang JQ, Chen HX, Yan ML, Huang L, et al. Hypoxia induces TWIST-activated epithelial-mesenchymal transition and proliferation of pancreatic cancer cells in vitro and in nude mice. Cancer Lett. 2016;383(1):73-84.

51. Zhu GH, Huang C, Feng ZZ, Lv XH, Qiu ZJ. Hypoxia-induced snail expression through transcriptional regulation by HIF-1alpha in pancreatic cancer cells. Dig Dis Sci. 2013;58(12):3503-15.

52. Deng SJ, Chen HY, Ye Z, Deng SC, Zhu S, Zeng Z, et al. Hypoxiainduced LnCRNA-BX111 promotes metastasis and progression of pancreatic cancer through regulating ZEB1 transcription. Oncogene. 2018;37(44):5811-28.

53. LuY, Ji N, Wei W, Sun W, Gong X, Wang X. MiR-142 modulates human pancreatic cancer proliferation and invasion by targeting hypoxiainducible factor 1 (HIF-1alpha) in the tumor microenvironments. Biol Open. 2017:6(2):252-9.

54. Li F, Tan H, Singh J, Yang J, Xia X, Bao J, et al. A 3D multiscale model of cancer stem cell in tumor development. BMC Syst Biol. 2013;7(Suppl 2):S12.

55. Zhou P, Li B, Liu F, Zhang M, Wang Q, Liu Y, et al. The epithelial to mesenchymal transition (EMT) and cancer stem cells: implication for treatment resistance in pancreatic cancer. Mol Cancer. 2017;16(1):52.

56. Hashimoto O, Shimizu K, Semba S, Chiba S, Ku Y, Yokozaki H, et al. Hypoxia induces tumor aggressiveness and the expansion of
CD133-positive cells in a hypoxia-inducible factor-1a-dependent manner in pancreatic cancer cells. Pathobiology. 2011;78(4):181-92.

57. Maeda K, Ding Q, Yoshimitsu M, Kuwahata T, Miyazaki Y, Tsukasa K, et al. CD133 modulate HIF-1alpha expression under hypoxia in EMT phenotype pancreatic cancer stem-like cells. Int J Mol Sci. 2016;17(7):1025.

58. Chen S, Zhang J, Chen J, Wang Y, Zhou S, Huang L, et al. RER1 enhances carcinogenesis and stemness of pancreatic cancer under hypoxic environment. J Exp Clin Cancer Res CR. 2019;38(1):15.

59. Zhu H, Wang D, Liu Y, Su Z, Zhang L, Chen F, et al. Role of the Hypoxiainducible factor-1 alpha induced autophagy in the conversion of nonstem pancreatic cancer cells into CD133 + pancreatic cancer stem-like cells. Cancer Cell Int. 2013;13(1):119.

60. Chiorean EG, Coveler AL. Pancreatic cancer: optimizing treatment options, new, and emerging targeted therapies. Drug Design Dev Ther. 2015;9:3529-45.

61. Wang F, Xia X, Yang C, Shen J, Mai J, Kim HC, et al. SMAD4 gene mutation renders pancreatic cancer resistance to radiotherapy through promotion of autophagy. Clin Cancer Res. 2018;24(13):3176-85.

62. Chand S, O'Hayer K, Blanco FF, Winter JM, Brody JR. The landscape of pancreatic cancer therapeutic resistance mechanisms. Int J Biol Sci. 2016;12(3):273-82.

63. Robey RW, Polgar O, Deeken J, To KW, Bates SE. ABCG2: determining its relevance in clinical drug resistance. Cancer Metastasis Rev. 2007:26(1):39-57.

64. He X, Wang J, Wei W, Shi M, Xin B, Zhang T, et al. Hypoxia regulates ABCG2 activity through the activation of ERK1/2/HIF-1alpha and contributes to chemoresistance in pancreatic cancer cells. Cancer Biol Ther. 2016;17(2):188-98.

65. Cheng ZX, Wang DW, Liu T, Liu WX, Xia WB, Xu J, et al. Effects of the HIF-1 $\mathrm{a}$ and NF-KB loop on epithelial-mesenchymal transition and chemoresistance induced by hypoxia in pancreatic cancer cells. Oncol Rep. 2014;31(4):1891-8.

66. Arora S, Bhardwaj A, Singh S, Srivastava SK, McClellan S, Nirodi CS, et al. An undesired effect of chemotherapy: gemcitabine promotes pancreatic cancer cell invasiveness through reactive oxygen species-dependent, nuclear factor KB- and hypoxia-inducible factor 1a-mediated up-regulation of CXCR4. J Biol Chem. 2013;288(29):21197-207.

67. Luo G, Xia X, Wang X, Zhang K, Cao J, Jiang T, et al. miR-301a plays a pivotal role in hypoxia-induced gemcitabine resistance in pancreatic cancer. Exp Cell Res. 2018;369(1):120-8.

68. Zhao T, Jin F, Xiao D, Wang H, Huang C, Wang X, et al. IL-37/STAT3/ HIF-1 a negative feedback signaling drives gemcitabine resistance in pancreatic cancer. Theranostics. 2020;10(9):4088-100.

69. Milanović D, Firat E, Grosu AL, Niedermann G. Increased radiosensitivity and radiothermosensitivity of human pancreatic MIA PaCa-2 and U251 glioblastoma cell lines treated with the novel Hsp90 inhibitor NVPHSP990. Radiat Oncol (London, England). 2013;8:42.

70. Nagaraju GP, Zakka KM, Landry JC, Shaib WL, Lesinski GB, El-Rayes BF. Inhibition of HSP90 overcomes resistance to chemotherapy and radiotherapy in pancreatic cancer. Int J Cancer. 2019;145(6):1529-37.

71. Huang C, Li Z, Li N, Li Y, Chang A, Zhao T, et al. Interleukin 35 expression correlates with microvessel density in pancreatic ductal adenocarcinoma, recruits monocytes, and promotes growth and angiogenesis of xenograft tumors in mice. Gastroenterology. 2018;154(3):675-88.

72. Zhang $W$, Xiong Z, Wei T, Li Q, Tan Y, Ling L, et al. Nuclear factor 90 promotes angiogenesis by regulating HIF-1 a/VEGF-A expression through the PI3K/Akt signaling pathway in human cervical cancer. Cell Death Dis. 2018;9(3):276

73. Azoitei N, Becher A, Steinestel K, Rouhi A, Diepold K, Genze F, et al. PKM2 promotes tumor angiogenesis by regulating HIF-1a through NF-KB activation. Mol Cancer. 2016;15:3.

74. Nagaraju GP, Park W, Wen J, Mahaseth H, Landry J, Farris AB, et al. Antiangiogenic effects of ganetespib in colorectal cancer mediated through inhibition of HIF-1a and STAT-3. Angiogenesis. 2013;16(4):903-17.

75. Lang SA, Moser C, Gaumann A, Klein D, Glockzin G, Popp FC, et al. Targeting heat shock protein 90 in pancreatic cancer impairs insulin-like growth factor-1 receptor signaling, disrupts an interleukin-6/signaltransducer and activator of transcription 3/hypoxia-inducible factor1alpha autocrine loop, and reduces orthotopic tumor growth. Clinical Cancer Res. 2007;13(21):6459-68. 
76. Liu Y, Steinestel K, Rouhi A, Armacki M, Diepold K, Chiosis G, et al. STK33 participates to HSP90-supported angiogenic program in hypoxic tumors by regulating HIF-1alpha/NEGF signaling pathway. Oncotarget. 2017;8(44):77474-88.

77. Tung KH, Lin CW, Kuo CC, Li LT, Kuo YH, Lin CW, et al. CHC promotes tumor growth and angiogenesis through regulation of HIF-1alpha and VEGF signaling. Cancer Lett. 2013;331(1):58-67.

78. Shan T, Ma J, Ma Q, Guo K, Guo J, Li X, et al. $\beta 2-A R-H I F-1$ a: a novel regulatory axis for stress-induced pancreatic tumor growth and angiogenesis. Curr Mol Med. 2013;13(6):1023-34.

79. Reeves $E$, James E. Antigen processing and immune regulation in the response to tumours. Immunology. 2017;150(1):16-24.

80. de Andrade FL, Tay RE, Pan D, Luoma AM, Ito Y, Badrinath S, et al. Antibody-mediated inhibition of MICA and MICB shedding promotes NK cell-driven tumor immunity. Science (New York, NY). 2018;359(6383):1537-42.

81. LuY, Hu J, Sun W, Duan X, Chen X. Hypoxia-mediated immune evasion of pancreatic carcinoma cells. Mol Med Rep. 2015;11(5):3666-72.

82. Ou ZL, Luo Z, Wei W, Liang S, Gao TL, Lu YB. Hypoxia-induced shedding of MICA and HIF1A-mediated immune escape of pancreatic cancer cells from NK cells: role of circ_0000977/miR-153 axis. RNA Biol. 2019;16(11):1592-603.

83. Takahashi K, Ehata S, Koinuma D, Morishita Y, Soda M, Mano H, et al. Pancreatic tumor microenvironment confers highly malignant properties on pancreatic cancer cells. Oncogene. 2018;37(21):2757-72.

84. Spivak-Kroizman TR, Hostetter G, Posner R, Aziz M, Hu C, Demeure MJ, et al. Hypoxia triggers hedgehog-mediated tumor-stromal interactions in pancreatic cancer. Cancer Res. 2013;73(11):3235-47.

85. Li N, Li Y, Li Z, Huang C, Yang Y, Lang M, et al. Hypoxia Inducible Factor 1 (HIF-1) recruits macrophage to activate pancreatic stellate cells in pancreatic ductal adenocarcinoma. Int J Mol Sci. 2016;17(6):799.

86. Katagiri T, Kobayashi M, Yoshimura M, Morinibu A, Itasaka S, Hiraoka $M$, et al. HIF-1 maintains a functional relationship between pancreatic cancer cells and stromal fibroblasts by upregulating expression and secretion of Sonic hedgehog. Oncotarget. 2018;9(12):10525-35.

87. Cho IR, Kaowinn S, Moon J, Soh J, Kang HY, Jung CR, et al. Oncotropic $\mathrm{H}-1$ parvovirus infection degrades HIF-1alpha protein in human pancreatic cancer cells independently of VHL and RACK1. Int J Oncol. 2015;46(5):2076-82.

88. Wilkes JG, O'Leary BR, Du J, Klinger AR, Sibenaller ZA, Doskey CM, et al. Pharmacologic ascorbate (P-AscH(-)) suppresses hypoxia-inducible factor-1alpha (HIF-1alpha) in pancreatic adenocarcinoma. Clin Exp Metas. 2018;35(1-2):37-51.

89. Sibenaller ZA, Welsh JL, Du C, Witmer JR, Schrock HE, Du J, et al. Extracellular superoxide dismutase suppresses hypoxia-inducible factor1alpha in pancreatic cancer. Free Radical Biol Med. 2014;69:357-66.

90. Chen H, Guan Y, Yuan G, Zhang Q, Jing N. A perylene derivative regulates HIF-1alpha and Stat3 signaling pathways. Bioorg Med Chem. 2014;22(4):1496-505.

91. Miyake $K$, Nishioka M, Imura S, Batmunkh E, Uto Y, Nagasawa H, et al. The novel hypoxic cytotoxin, TX-2098 has antitumor effect in pancreatic cancer; possible mechanism through inhibiting VEGF and hypoxia inducible factor-1alpha targeted gene expression. Exp Cell Res. 2012;318(13):1554-63.

92. Tang LR, WU JX, Cai SL, Huang YX, Zhang XQ, Fu WK, et al. Prolyl hydroxylase domain 3 influences the radiotherapy efficacy of pancreatic cancer cells by targeting hypoxia-inducible factor-1a. Onco Targets Ther. 2018;11:8507-15.

93. Lv C, Kong H, Dong G, Liu L, Tong K, Sun H, et al. Antitumor efficacy of a-solanine against pancreatic cancer in vitro and in vivo. PLoS ONE. 2014;9(2):e87868.

94. Wen Z, Huang C, Xu Y, Xiao Y, Tang L, Dai J, et al. a-Solanine inhibits vascular endothelial growth factor expression by down-regulating the ERK1/2-HIF-1 $\mathrm{a}$ and STAT3 signaling pathways. Eur J Pharmacol. 2016;771:93-8.

95. Seo JH, Jung KH, Son MK, Yan HH, Ryu YL, Kim J, et al. Anti-cancer effect of HS-345, a new tropomyosin-related kinase A inhibitor, on human pancreatic cancer. Cancer Lett. 2013;338(2):271-81.

96. Ryu YL, Jung KH, Son MK, Yan HH, Kim SJ, Shin S, et al. Anticancer activity of HS-527, a novel inhibitor targeting PI3-kinase in human pancreatic cancer cells. Cancer Lett. 2014;353(1):68-77.

97. Nagaraju GP, Zhu S, Ko JE, Ashritha N, Kandimalla R, Snyder JP, et al. Antiangiogenic effects of a novel synthetic curcumin analogue in pancreatic cancer. Cancer Lett. 2015;357(2):557-65.

98. Niu F, Li Y, Lai FF, Ni L, Ji M, Jin J, et al. LB-1 exerts antitumor activity in pancreatic cancer by inhibiting HIF-1alpha and Stat3 signaling. J Cell Physiol. 2015;230(9):2212-23.

99. Zhao T, Ren H, Jia L, Chen J, Xin W, Yan F, et al. Inhibition of HIF-1a by PX-478 enhances the anti-tumor effect of gemcitabine by inducing immunogenic cell death in pancreatic ductal adenocarcinoma. Oncotarget. 2015;6(4):2250-62.

100. Schwartz DL, Bankson JA, Lemos R Jr, Lai SY, Thittai AK, He Y, et al. Radiosensitization and stromal imaging response correlates for the HIF-1 inhibitor PX-478 given with or without chemotherapy in pancreatic cancer. Mol Cancer Ther. 2010;9(7):2057-67.

101. Yasui H, Matsumoto S, Devasahayam N, Munasinghe JP, Choudhuri R, Saito K, et al. Low-field magnetic resonance imaging to visualize chronic and cycling hypoxia in tumor-bearing mice. Cancer Res. 2010;70(16):6427-36.

102. Dewhirst MW. Relationships between cycling hypoxia, HIF-1, angiogenesis and oxidative stress. Radiat Res. 2009;172(6):653-65.

103. Olbryt M, Habryka A, Student S, Jarząb M, Tyszkiewicz T, Lisowska KM. Global gene expression profiling in three tumor cell lines subjected to experimental cycling and chronic hypoxia. PLoS ONE. 2014;9(8):e105104.

\section{Publisher's Note}

Springer Nature remains neutral with regard to jurisdictional claims in published maps and institutional affiliations.

Ready to submit your research? Choose BMC and benefit from:

- fast, convenient online submission

- thorough peer review by experienced researchers in your field

- rapid publication on acceptance

- support for research data, including large and complex data types

- gold Open Access which fosters wider collaboration and increased citations

- maximum visibility for your research: over $100 \mathrm{M}$ website views per year

At BMC, research is always in progress.

Learn more biomedcentral.com/submissions 